\title{
CIRCULAR SUMMATION AND LOCALIZATION OF DOUBLE TRIGONOMETRIC SERIES
}

\author{
BY \\ LEONARD D. BERKOVITZ
}

In this paper we shall study the problem of localization in the case of double trigonometric series summed by the method of circular summation introduced by Bochner in his treatment of multiple Fourier series [1] (1). To develop our theory of localization we shall use the process of formal multiplication of series in a manner analogous to that employed by Rajchman and Zygmund [6] in their investigations of localization phenomena exhibited by trigonometric series in one variable. However, we shall apply these ideas to circular sums and means. We also extend our results to higher dimensions and consider briefly types of summation defined by certain convex curves.

The author would like to take this opportunity to thank Professor Antoni Zygmund for suggesting this problem and for his guidance and encouragement.

1. Definitions and notation. A double trigonometric series is a series of the form

$$
T=\sum_{m, n=-\infty}^{\infty} c_{m n} e^{i(m x+n y)},
$$

where the numbers $c_{m n}$ are arbitrary complex numbers. Such a series is said to converge in the ordinary, or Pringsheim, sense to a limit $L$ at the point $(x, y)$, if the sequence of partial sums $S_{M N}$ defined by

$$
S_{M N}=\sum_{m, n=-M,-N}^{M, N} c_{m, n} e^{i(m x+n y)}
$$

satisfies the following condition. Given an $\epsilon>0$ we can find integers $N_{0}$ and $M_{0}$ such that whenever $N>N_{0}$ and $M>M_{0}$ we have $\left|S_{M N}-L\right|<\epsilon$. We say that the series converges rapidly to a limit $L$ in case $\left|S_{M N}-L\right|<\epsilon$ for all but a finite number of indices.

As was already suggested, we shall primarily be dealing with a third method of summation, namely circular summation. To this end we adopt the following conventions. An index pair will be denoted by the capital letter of the first index occurring. For example, $(m, n)$ will be denoted simply by $M$. By $|M|$ we shall mean the distance of the lattice point $(m, n)$ from the origin. Thus $|M|=\left(m^{2}+n^{2}\right)^{1 / 2}$. Furthermore, we shall write the expression

Presented to the Society, April 28, 1950; received by the editors August 3, 1950.

(') Numbers in brackets refer to the bibliography at the end of the paper. 
$(m x+n y)$ simply as $M X$. In the event that the limits of summation are $\pm \infty$ and there is no doubt as to the index of summation, we shall drop the indices under the summation sign. Should several pairs of indices be involved, then we shall merely indicate by the appropriate capital letter which pair is to be summed over, with the limits again omitted if they are $\pm \infty$. In practically all situations arising, no confusion will result from this convention. In any case where there is a possibility of confusion, our convention will be dropped and everything will be spelled out in the usual way. Our series $T$ can now be written as

$$
T=\sum c_{M} e^{i M X} .
$$

With these preliminaries attended to, we proceed to discuss circular summation. By the circular partial sums $S_{R}(x, y)$ of the series $T$, we mean the numbers defined by

$$
S_{R}(x, y)=\sum_{|M| \leqq R} c_{M} e^{i M X}
$$

where $R$ is a continuous parameter. We say that $T$ is circularly summable to the value $L(x, y)$ at the point $(x, y)$ in case $\lim _{R \rightarrow \infty} S_{R}(x, y)=L(x, y)$. Clearly, the case $x=0, y=0$ is the case of numerical series. A series will be said to be absolutely circularly summable if the series obtained by replacing each term by its absolute value is circularly summable. It is quite evident that a series is absolutely circularly summable if and only if the series of absolute values is convergent in the ordinary sense. For such series the ordinary and circular methods are equivalent.

The series $T$ will be said to be circularly summable $(C, \eta), \eta>0$, to sum $L(x, y)$ in case

$$
\lim _{R \rightarrow \infty} \bar{\sigma}_{R}^{\eta}(x, y)=L(x, y)
$$

where

$$
\bar{\sigma}_{R}^{\eta}(x, y)=\frac{\eta}{R^{\eta}} \int_{0}^{R} S_{r}(x, y)(R-r)^{\eta-1} d r=\sum_{|M| \leqq R} c_{M} e^{i M X}\left(1-\frac{|M|}{R}\right)^{\eta} .
$$

The numbers $\bar{\sigma}_{R}^{\eta}(x, y)$ are called the $(C, \eta)$ means of rank $R$ of the series $T$. From the second formula for $\bar{\sigma}_{R}^{\eta}(x, y)$, we see that it is natural to define summability $(C, 0)$ as ordinary circular summation. It can be shown [3, p. 29] that summability $(C, \eta)$ implies summability $(C, \eta+\epsilon), \epsilon>0$. A theorem of Hardy [2] tells us that if we were to define the $(C, \eta)$ circular means as

$$
\sigma_{R}^{\eta}(x, y)=\frac{2 \eta}{R^{2 \eta}} \int_{0}^{R} S_{r}(x, y)\left(R^{2}-r^{2}\right)^{\eta-1} r d r=\sum_{|M| \leqq R} c_{M} e^{i \dot{M X}}\left(1-\frac{|M|^{2}}{R^{2}}\right)^{\eta},
$$


then $\bar{\sigma}_{R}^{\eta}(x, y)-L(x, y)=o(1)$ if and only if $\sigma_{R}^{\eta}(x, y)-L(x, y)$ is $o(1)$. An examination of the proof of this theorem, at least for $\eta$ an integer, shows that in our case $\bar{\sigma}_{R}^{\eta}(x, y)-L(x, y)$ is $o\left(R^{-\epsilon}\right), \epsilon>0$, if and only if $\sigma_{R}^{\eta}(x, y)-L(x, y)$ $=o\left(R^{-\epsilon}\right)$. See $[2, \mathrm{p} .76]\left({ }^{2}\right)$. We shall make use of this strong equivalence and in any given situation shall take whichever definition is more convenient at the moment.

In the sequel we shall occasionally drop the adjective "circular" when speaking of circular summation. However, when speaking of circular processes we shall use the words "summation," "summable," or "summability," in contrast to the word "convergence" which shall be reserved for the usual concepts. Thus our intent will be clear.

2. Formal multiplication. We begin by developing the theory of formal multiplication as far as we shall need it. Let

$$
T_{1}=\sum a_{M} e^{i M X} \text { and } T_{2}=\sum \alpha_{M} e^{i M X}
$$

be two double trigonometric series. Their formal product, when it exists, is defined to be the series

$$
T_{3}=\sum A_{M} e^{i M X}
$$

where

$$
A_{M}=\sum_{P} a_{P} \alpha_{M \rightarrow P}
$$

If the numbers $a_{M}$ are bounded and the series $\sum\left|\alpha_{M}\right|$ is convergent, then the numbers $A_{M}$ are defined. Hence the formal product exists. In fact we have the following lemma concerning the order of the coefficients $A_{M}$.

Lemma 1. Suppose that $T_{1}$ is a series with coefficients $o(|M| \gamma), \gamma \geqq 0$, and suppose that $T_{2}$ is a series such that $\sum\left|\alpha_{M}\right||M| \gamma$ is convergent. Then the series (1) converges and $A_{M}$ is $o(|M| \gamma)$. If $\gamma=0$ the condition is to be taken as o(1) as $|M| \rightarrow \infty$.

\section{Write}

$$
\begin{aligned}
A_{M} & =\sum_{P} a_{P} \alpha_{M-P}=\left(\sum_{|P|<|M| / 2}+\sum_{|M| / 2 \leqq|P| \leqq 2|M|}+\sum_{|P|>2|M|}\right) a_{P} \alpha_{M-P} \\
& =\sum_{1}+\sum_{2}+\sum_{3} .
\end{aligned}
$$

But

$$
\left|\sum_{1}\right| \leqq K|M|^{\gamma} \sum_{|P|<|M| / 2}\left|\alpha_{M-P}\right|=o(1) K|M|^{\gamma}
$$

where $K$ is a positive constant and $o(1)$ is to be taken as $o(1)$ as $|M| \rightarrow \infty$.

(2) In Hardy's notation we have $\lambda_{k}=n_{k}$, where the sequence $\left\{n_{k}\right\}$ consists of those integers representable as the sum of two squares, $\mu_{k}=\left(n_{k}\right)^{1 / 2}$, and so $\mu=\sigma^{1 / 2}, \zeta=\eta^{1 / 2}$. 
Moreover,

$$
\begin{aligned}
\left|\sum_{2}\right| & \leqq o(|M| \gamma) \sum\left|\alpha_{M}\right|=o(|M| \gamma), \\
\left|\sum_{3}\right| & \leqq o\left\{\sum_{|P|>2|M|}\left(\frac{|P|}{|M-P|}\right)^{\gamma}\left|\alpha_{M-P}\right||M-P|^{\gamma}\right\} \\
& =o\left\{2^{\gamma} \sum_{|P|>2|M|}\left|\alpha_{M-P}\right||M-P|^{\gamma}\right\}=o(1) .
\end{aligned}
$$

Combining the estimates on $\sum_{1}, \sum_{2}$, and $\sum_{3}$, we establish the lemma.

Having determined conditions under which the formal product exists, we now prove some theorems about its behavior.

THEOREM 1. Let $T_{1}$ and $T_{2}$ be two double trigonometric series with the following properties:

$$
\begin{gathered}
a_{M}=o\left(|M|^{-1}\right), \\
\alpha_{M}=O\left(|M|^{-5}\right), \\
\sum \alpha_{M} e^{i M X}=0
\end{gathered}
$$

for $(x, y)$ belonging to a set $E$ in the plane. Then the series $T_{3}$ whose terms $A_{M}$ are given by (1) is the formal product of $T_{1}$ and $T_{2}$ and is circularly summable to zero, uniformly for all $(x, y)$ in $E$.

In the proof several estimates arise quite naturally, and since they will be needed for later theorems, we shall state them as separate lemmas.

LEMma 2. The number of lattice points in a circle of radius $R$ is

$$
\pi R^{2}+O(R) \text {. }
$$

This is a very well known and elementary result.

Lemma 3. Let $T_{2}$ be a series satisfying the conditions of the theorem, except for (ii) which now reads

$$
\alpha_{M}=O\left(|M|^{-\theta}\right),
$$

Then for any $(x, y)$ in $E$, any positive real number $R$, and any point $P$ whose norm does not satisfy the inequality $R-1 \leqq|P| \leqq R+1$

$$
\begin{aligned}
\sum_{|M|>R}\left|\alpha_{M}\right| & =O\left(R^{-(\theta-2)}\right), \\
\left|\sum_{|M| \leqq R} \alpha_{M-P} e^{i(M-P) X}\right| & =O\left(|R-| P||^{-(\theta-2)}\right) .
\end{aligned}
$$

To establish (3), we first divide the region exterior to the circle of radius $R$ into annuli $A_{j}, j=0,1,2, \cdots$, each of width one. Denote the number of lattice points in the annulus $A_{j}$ by $L_{j}$. Then in view of (2) 


$$
\begin{aligned}
L_{j} & \leqq \pi\left\{(R+j+1)^{2}-(R+j)^{2}\right\}+O(R+j+1) \\
& =\pi\{2 R+2 j+1\}+O(R+j+1) .
\end{aligned}
$$

Hence we may write

$$
\sum_{|M|>R}\left|\alpha_{M}\right|=\sum_{j=0}^{\infty} \sum_{M \in A_{j}}\left|\alpha_{M}\right| \leqq O\left\{\sum_{j=0}^{\infty} \frac{R+j+1}{(R+j)^{\theta}}\right\}=O\left(\frac{1}{R^{\theta-2}}\right)
$$

which is (3).

Once we have (3) it is an easy matter to obtain (4). Suppose first $|P|>R$ +1 . If we construct a circle $C_{1}$ of radius $R$ with $P$ as center, then this circle will be tangent to the circle $C_{2}$ with center at the origin and radius $|P|-R$. Also, $C_{1}$ will be exterior to $C_{2}$. Hence

$$
\begin{aligned}
\left|\sum_{|M| \leqq R} \alpha_{M \rightarrow P} e^{i(M-P) X}\right| & \leqq \sum_{|M| \leqq R}\left|\alpha_{M-P}\right| \leqq \sum_{|M|>|P|-R}\left|\alpha_{M}\right| \\
& =O\left(\frac{1}{(|P|-R)^{\theta-2}}\right) .
\end{aligned}
$$

On the other hand, if $|P|<R-1$ we again construct a circle $C_{1}$ of radius $R$ with $P$ as center. $C_{1}$ will be tangent to the circle $C_{2}$ whose center is at the origin and whose radius is $R-|P|$. Further, $C_{1}$ will contain $C_{2}$. Making use of condition (iii), we can say

$$
\left|\sum_{|M| \leqq R} \alpha_{M-P} e^{i(M-P) X}\right|=\left|\sum_{|M|>R} \alpha_{M-P} e^{i(M-P) X}\right|
$$

and by (3) this is $O\left((R-|P|)^{-(\theta-2)}\right)$. The lemma is thus proved.

We now take up the proof of the theorem proper. The fact that the formal product is well defined follows either from Lemma 1 or the remark immediately preceding the lemma. If we denote the circular partial sums of the product by $S_{R}(x, y)$, we must show that $\lim _{R \rightarrow \infty} S_{R}(x, y)=0$, uniformly for all $(x, y)$ in $E$. For simplicity of notation we shall assume that the set $E$ consists of the point $(o, o) . S_{R}(o, o)$ will be written simply as $S_{R}$. From the method of proof it will be clear that the result is valid for any point $(x, y)$, and furthermore, if $E$ does not reduce to a point, the convergence of $S_{R}(x, y)$ on $E$ is uniform.

We write

$$
\begin{aligned}
S_{R} & =\sum_{|M| \leqq R} A_{M}=\sum_{|M| \leqq R} \sum_{P} a_{P} \alpha_{M-P}=\sum_{P} a_{P} \sum_{|M| \leqq R} \alpha_{M-P} \\
& =\left(\sum_{|P| \leqq R / 2}+\sum_{R / 2<|P|<3 R / 2}+\sum_{|P| \leqq 3 R / 2}\right) \sum_{|M| \leqq R} \alpha_{M-P}=A+B+C .
\end{aligned}
$$

Each of the sums $A, B$, and $C$ will be shown to approach zero as $R$ tends to 
infinity. Sum $A$ is dealt with quite readily as follows.

$$
|A| \leqq \sum_{|P| \leqq R / 2}\left|a_{P}\right|\left|\sum_{|M| \leqq R} \alpha_{M-P}\right|=\sum_{|P| \leqq R / 2}\left|a_{P}\right|\left|\sum_{|M|>R} \alpha_{M-P}\right|
$$

since $\sum \alpha_{M}=0$. Therefore

$$
|A| \leqq \sum_{|P| \leqq R / 2}\left|a_{P}\right| \sum_{|M|>R / 2}\left|\alpha_{M}\right| .
$$

In view of the last inequality, statements (2) and (3), and the fact that the coefficients $a_{P}$ are bounded, we have

$$
|A|=O\left(R^{2}\right) O\left(\frac{1}{R^{3}}\right)=O\left(\frac{1}{R}\right) .
$$

This is the desired result.

$C$ is treated in a manner similar to that used to establish (3). With the notation set up there and with the aid of (4) and (5), we see that

$$
\begin{aligned}
|C| & \leqq \sum_{j=0}^{\infty}\left(\sum_{P \in A_{j}}\left|a_{P}\right|\left|\sum_{|M| \leqq R} \alpha_{M-P}\right|\right) \\
& \leqq O\left\{\sum_{j=0}^{\infty} \frac{R+j+1}{(3 R / 2+j-R)^{3}}\right\}=O\left(\frac{1}{R}\right) .
\end{aligned}
$$

REMARK 1 . It should be noted that only the fact that $a_{P}=O(1)$ was required of $T_{1}$ to get the convergence to zero of $A$ and $C$.

Finally we dispose of $B$.

$$
\begin{aligned}
B & =\left(\sum_{R / 2<|P|<R-1}+\sum_{R-1 \leqq|P| \leqq R+1}+\sum_{R+1<|P|<3 R / 2} a_{P}\right) \sum_{|M| \leqq R} \alpha_{M-P} \\
& =B_{1}+B_{2}+B_{3} .
\end{aligned}
$$

Using (2), the hypothesis

$$
a_{P}=o\left(|P|^{-1}\right),
$$

and the absolute convergence of $T_{2}$ we immediately see that

$$
\left|B_{2}\right| \leqq o(1)\left\{\frac{\pi\left[(R+1)^{2}-(R-1)^{2}\right]+O(R+1)}{(R-1)}\right\} o(1)=o(1) .
$$

To evaluate $B_{3}$, subdivide the annulus formed by the circles whose radii are $R+1$ and $3 R / 2$ by drawing circles with centers at the origin and radii $(R+1)+j, j=1,2, \cdots, j_{R}$, where $j_{R}=[3 R / 2-(R+1)]$. We thus get annuli $A_{j}, j=1,2, \cdots, j_{R}$, of width one and usually an annulus $A_{j_{R}+1}$ whose width 
is less than one. Clearly,

$$
\left|B_{3}\right| \leqq \sum_{j=1}^{(*)}\left(\sum_{P \in A_{j}}\left|a_{P}\right|\left|\sum_{|M| \leqq R} \alpha_{M-P}\right|\right)
$$

where $(*)=j_{R}+1$ if the annulus $A_{j_{R}+1}$ exists and $(*)=j_{R}$ otherwise. From (2), (4), and the fact that $a_{P}=o\left(|P|^{-1}\right)$, we obtain that the preceding series is majorized by

$$
o(1) O\left\{\sum_{j=1}^{(*)} \frac{(R+j)^{2}-(R+j-1)^{2}+(R+j)}{(R+j)(j)^{3}}\right\}=o\left\{\sum_{j=1}^{(*)} \frac{1}{(j)^{3}}\right\}=o(1) .
$$

Similarly we can show that $B_{1}$ is $o(1)$ and so complete the proof of Theorem 1.

We observe at this point that the condition (ii) of the theorem could have been replaced by the more economical one of $\alpha_{M}=O\left(|M|^{-(4+\epsilon)}\right), \epsilon>0$, or by $\sum\left|\alpha_{M}\right||M|^{3}<\infty$.

If the series $T_{2}$ converges to a function $\lambda(x, y)$ which need not be zero, then we have the following result.

THEOREM $1^{\prime}$. Let $T_{1}$ and $T_{2}$ be two series satisfying the conditions of Theorem 1 , except that $T_{2}$ converges to a function $\lambda(x, y)$ which need not be zero. Then

$$
T_{3}-\lambda T_{1}=\sum A_{M} e^{i M X}-\lambda(x, y) \sum a_{M} e^{i M X}
$$

is circularly summable to zero, uniformly for $(x, y)$ in the fundamental square $\Omega$ bounded by the lines $x=0, x=2 \pi, y=0, y=2 \pi$.

We consider the series

$$
T_{2}^{*}=\sum \alpha_{M}^{*} e^{i M X} \quad \text { where }\left\{\begin{array}{l}
\alpha_{M}^{*}=\alpha_{M} \quad \text { if } \quad|M| \neq 0, \\
\alpha_{00}^{*}=\alpha_{00}-\lambda(x, y) .
\end{array}\right.
$$

The series $T_{2}^{*}$ has coefficients which depend on $(x, y)$ but which satisfy the conditions put on the coefficients of $T_{2}$ in Theorem 1 uniformly for $(x, y)$ in $\Omega$. Also $T_{2}^{*}$ converges to zero in $\Omega$. An examination of the proof of Theorem 1 will show that if the coefficients $\alpha_{M}$ depend on $(x, y)$ and the required conditions on the $\alpha_{M}$ are satisfied uniformly for $(x, y)$ in $\Omega$, then $T_{3}$ is still circularly summable to zero, uniformly on $E$. Letting $T_{3}^{*}=T_{1} T_{2}^{*}$ and applying the above discussion to $T_{3}^{*}$ yields the fact that $T_{3}^{*}$ is uniformly circularly summable to zero on $\Omega$.

But

$$
T_{3}^{*}=\sum A_{M}^{*} e^{i M X}
$$

where 


$$
\begin{aligned}
A_{M}^{*} & =\sum a_{M} \alpha_{M-P}^{*}=\sum_{P \neq M} a_{M} \alpha_{M-P}+a_{M}\left(\alpha_{00}-\lambda(x, y)\right) \\
& =\sum_{P} a_{M} \alpha_{M-P}-\lambda(x, y) a_{M}=A_{M}-\lambda(x, y) a_{M} .
\end{aligned}
$$

Hence the theorem follows.

With Theorem 1 as base point we shall consider the behavior of the formal product of $T_{1}$ and $T_{2}$ when we no longer assume $a_{M}=o\left(|M|^{-1}\right)$, but merely $a_{M}=o(|M| \gamma), \gamma \geqq-1$. In order to do this we must first define three operators, $\partial / \partial x, \partial / \partial y$, and $\nabla^{2}$, which in effect consist of formal partial differentiation of series and formal forming of Laplacians. Let

Then

$$
T=\sum C_{M} e^{i M X} \text {. }
$$

$$
\begin{gathered}
\frac{\partial T}{\partial x}=\sum i m c_{M} e^{i M X}, \quad \frac{\partial T}{\partial y}=\sum i n c_{M} e^{i M X}, \\
\nabla^{2} T=-\sum|M|{ }^{2} c_{M} e^{i M X} .
\end{gathered}
$$

By the symbol $\partial^{(r)} / \partial x^{(r)}, r$ an integer greater than 0 , we shall mean the operator $\partial / \partial x$ applied $r$ times in succession, and similarly for the operators $\partial^{(r)} / \partial y^{(r)}$ and $\left(\nabla^{2}\right)^{(r)}$. The operators $\partial^{(0)} / \partial x^{(0)}, \partial^{(0)} / \partial y^{(0)}$, and $\left(\nabla^{2}\right)^{(0)}$ shall be interpreted as the identity operators. It is clear that these operators and their powers commute with each other in all possible ways. Our theorem can now be stated.

THEOREM 2. Let $T_{1}$ be a double trigonometric series with coefficients o $(|M| \gamma)$, $\gamma \geqq-1$. Let $T_{2}$ be a trigonometric series with coefficients $O\left(|M|^{-\delta}\right)$, where $\delta=5$ $+2(\bar{\gamma}+1)$ and $\bar{\gamma}$ is the smallest integer greater than or equal to $\gamma$. Finally, suppose that

$$
\frac{\partial^{(r)}}{\partial x^{(r)}} \frac{\partial^{(s)}}{\partial y^{(s)}}\left(\nabla^{2}\right)^{(t)} T_{2}=0
$$

for all triples of integers $(r, s, t)$ satisfying the condition $r+s+t \leqq \bar{\gamma}+1$, and for all $(x, y)$ in a set $E\left({ }^{3}\right)$. Then $T_{3}$, the formal product of $T_{1}$ and $T_{2}$, is defined and is circularly summable $(C, \gamma+1)$ to zero, uniformly for all $(x, y)$ in $E$.

As in the proof of Theorem 1, we shall assume that $E$ consists of the origin. The method of proof will again show the result to be valid for any point, and if $E$ does not reduce to a point, the convergence of $\sigma_{R}^{\gamma+1}(x, y)$ on $E$ is uniform.

The fact that the formal product is defined is a consequence of Lemma 1,

(3) For example, a series all of whose derivatives up to and including those of order $2(\gamma+1)$ vanish on $E$ satisfies this condition. 
and the major task is to show that

$$
\sigma_{R}^{\gamma+1}=\frac{2(\gamma+1)}{R^{2(\gamma+1)}} \int_{0}^{R} S(r)\left(R^{2}-r^{2}\right)^{\gamma} r d r=o(1)
$$

where $S(r)=S_{r}$.

First we shall prove that the theorem is true for the case that $\gamma$ is an integer greater than or equal to -1 . For convenience of terminology we shall consider zero to be such an integer. Then

$$
\begin{aligned}
\sigma_{R}^{\gamma+1} & =\sum_{|M| \leqq R} A_{M}\left(1-\frac{|M|^{2}}{R^{2}}\right)^{\gamma+1}=\sum_{P} a_{P} \sum_{|M| \leqq R} \alpha_{M-P}\left(1-\frac{|M|^{2}}{R^{2}}\right)^{\gamma+1} \\
& =\left(\sum_{|P|<3 R / 2}+\sum_{|P| \geqq 3 R / 2} a_{P}\right) \sum_{|M| \leqq R} \alpha_{M-P}\left(1-\frac{|M|^{2}}{R^{2}}\right)^{\gamma+1}=A^{\prime}+B^{\prime} .
\end{aligned}
$$

We now show that $B^{\prime}=o(1)$ as $|M| \rightarrow \infty$. Taking into account the fact that $0 \leqq\left(1-|M|^{2} / R^{2}\right) \leqq 1$, statement (3), and the order of the coefficients of $T_{1}$, we get

$$
\begin{aligned}
\left|B^{\prime}\right| & \leqq \sum_{|P| \geqq 3 R / 2}\left|a_{P}\right| \sum_{|M| \leqq R}\left|\alpha_{M-P}\right| \\
& =O\left\{\sum_{|P| \geqq 3 R / 2}\left(\frac{|P|}{|M-P|}\right)^{\gamma} \frac{1}{(|P|-R)^{3+\zeta}}\right\} \\
& =O\left\{\sum_{|P| \geqq 3 R / 2} \frac{3^{\gamma}}{(|P|-R)^{3+\zeta}}\right\}
\end{aligned}
$$

where $\zeta=2(\bar{\gamma}+1)-\gamma$. The methods used to prove that sum $C$ in (7) was $O(1 / R)$ can now be used to show that the sum on the right is $O\left(1 / R^{1+\zeta}\right)$.

REMARK 2. The condition $a_{P}=o\left(|M|^{\gamma}\right)$ on the coefficients of $T_{1}$ could have been replaced by $O(|M| \gamma)$ and the result just proved would still be valid.

REMARK 3. In this argument we did not use the fact that $\gamma$ was an integer.

Lemma 4 , which follows, tells us that $A^{\prime}$ is $o(1)$ and so completes the proof of the theorem if $\gamma$ is an integer.

LEMмA 4. Under the hypotheses of the theorem,

$$
\sum_{|P|<3 R / 2} a_{P} \sum_{|M| \leqq R} \alpha_{M \rightarrow P}\left(1-\frac{|M|^{2}}{R^{2}}\right)^{\gamma+1}=o(1), \quad \gamma=-1,0,1,2, \cdots .
$$

For $\gamma=-1$ this was proved in Theorem 1 . Suppose now that $\gamma>-1$. In view of the equation

(9) $m^{2}+n^{2}=p^{2}+q^{2}+(m-p)^{2}+(n-q)^{2}-2 i(m-p) i p-2 i(n-q) i q$, 
we may write

$$
\begin{aligned}
A^{\prime}= & \sum_{|P|<3 R / 2} \frac{a_{P}}{R^{2}} \sum_{|M| \leqq R} \alpha_{M-P}\left(R^{2}-|M|^{2}\right)\left(1-\frac{|M|^{2}}{R^{2}}\right)^{\gamma} \\
= & \sum_{|P|<3 R / 2} \frac{a_{P}}{R^{2}} \sum_{|M| \leqq R} \alpha_{M-P}\left(-|M-P|^{2}\right)\left(1-\frac{|M|^{2}}{R^{2}}\right)^{\gamma} \\
& +2 i \sum_{|P|<3 R / 2} \frac{p a_{P}}{R^{2}} \sum_{|M| \leqq R} \alpha_{M-P} i(m-p)\left(1-\frac{|M|^{2}}{R^{2}}\right)^{\gamma} \\
& +2 i \sum_{|P|<3 R / 2} \frac{q a_{P}}{R^{2}} \sum_{|M| \leqq R} \alpha_{M-P} i(n-q)\left(1-\frac{|M|^{2}}{R^{2}}\right)^{\gamma} \\
& +\sum_{|P|<3 R / 2} \frac{a_{P}\left(R^{2}-|P|^{2}\right)}{R^{2}} \sum_{|M| \leqq R} \alpha_{M-P}\left(1-\frac{|M|^{2}}{R^{2}}\right)^{\gamma} \\
= & S_{1}+S_{2}+S_{3}+S_{4} .
\end{aligned}
$$

To each of the sums $S_{1}, S_{2}, S_{3}, S_{4}$, we repeat the process of factoring out $\left(R^{2}-|M|^{2}\right) / R^{2}$ and applying the decomposition (9) to the numerator. In $\gamma+1$ steps we shall obtain $4^{\gamma+1}$ sums of the form

$$
\sum_{\xi}=\sum_{|P|<3 R / 2} \frac{a_{P} p^{\lambda(\xi)} q^{\mu(\xi)}\left(R^{2}-|P|^{2}\right)^{\nu(\xi)}}{R^{2 \gamma+2}} \sum_{|M| \leqq R} \beta_{M-F}^{(\xi)}
$$

where:

(a) $\xi=1,2,3, \cdots, 4^{\gamma+1}$,

(b) $\lambda(\xi), \mu(\xi)$, and $\nu(\xi)$ are integers depending on $\xi$ and which satisfy the relation $\lambda(\xi)+\mu(\xi)+\nu(\xi) \leqq \gamma+1$, for all $\xi$. Henceforth, we shall merely write $\lambda, \mu, \nu$ and drop the argument $\xi$.

At this point it should be remarked that the $4^{\gamma+1}$ sums $\sum_{\xi}$ are not all distinct. This, however, need not concern us.

For every $\xi$, the series $\sum \beta_{M}^{(\xi)}$ can be considered as being obtained from $T_{2}$ by application of the operators $\partial / \partial x, \partial / \partial y, \nabla^{2}$, and their powers. Now we know that in getting $\sum \beta_{M}^{(\xi)}$ from $T_{2}$ we applied the partial derivative operators $\lambda+\mu$ times. If we denote by $\tau$ the number of times we applied the Laplacian operator, we see that for all $\xi$,

$$
\lambda+\mu+\nu+\tau=\gamma+1
$$

and so by (7), $\sum \beta_{M}^{(\xi)}=0$. Recalling the fact that the series $T_{2}$ has coefficients $O\left(|M|^{-\delta}\right)$, we note that every application of a partial derivative operator to $T_{2}$ will diminish $\delta$ by one, and every application of the Laplacian operator will diminish $\delta$ by two. Therefore, $\beta_{M}^{(\xi)}=O\left(|M|^{-\eta}\right)$, where

$$
\eta=5+2(\gamma+1)-(\lambda+\mu+2 \tau)=5+\lambda+\mu+2 \nu .
$$

Combining these results with (10) and applying (4) yields 


$$
\begin{aligned}
&\left|\sum_{\xi}\right|= O\left\{\sum_{|P|<3 R / 2}^{\prime} \frac{\left|a_{P}\right|}{R^{\gamma+1}} \frac{(|R-| P|||R+| P||)^{\prime}}{R^{\gamma+1-(\lambda+\mu)}} \frac{1}{(R-|P|)^{n-2}}\right\} \\
&+O\left\{\sum_{R-1 \leqq|P| \leqq R+1} \frac{\left|a_{P}\right|}{R^{\gamma+1}} \frac{(|R-| P|||R+| P||)^{\nu}}{R^{\gamma+1-(\lambda+\mu)}}\right\} \\
& \leqq \\
&\left.O \sum_{|P|<3 R / 2}^{\prime} \frac{\left|a_{P}\right|}{R^{\gamma+1}} \frac{1}{|R-| P||^{3+\lambda+\mu+\nu}}\right\} \\
&+O\left\{\sum_{R-1 \leqq|P| \leqq R+1} \frac{\left|a_{P}\right|}{R^{\gamma+1}}\right\}
\end{aligned}
$$

where the mark' indicates that we are to omit from consideration those indices which satisfy $R-1 \leqq|P| \leqq R+1$. Since $\left|a_{P}\right| / R^{\gamma+1}=o\left(|P|^{\gamma / R^{\gamma+1}}\right)$ and $|P|<3 R / 2$, we see that (11) is essentially the same as the sum $A+B$ of equation (6) in Theorem $\left.1{ }^{4}\right)$. But we have already shown there that $A$ and $B$ are both $o(1)$, and hence we have shown that for all $\xi, \sum_{\xi}$ is $o(1)$. This completes the proof of the lemma.

REMARK 4. At this stage we note that if $a_{M}=o(|M| \gamma), k-1<\gamma<k$, where $k$ is an integer greater than or equal to zero, we may put $\gamma+\epsilon=k, 0<\epsilon<1$, and say $a_{M}=o\left(|M|^{k-\epsilon}\right)$. The formal product will then be summable $(C, k+1)$ to zero, and an inspection of the above proof informs us that $\sigma_{R}^{k+1}=o\left(R^{-\epsilon}\right)$. We have already seen in $\$ 1$ that Hardy's theorem [2] implies that if $\sigma_{R}^{k+1}$ $=o\left(R^{-\epsilon}\right)$ then

$$
\bar{\sigma}_{R}^{k+1}=\frac{k+1}{R^{k+1}} \int_{0}^{R} S(r)(R-r)^{k} d r=o\left(R^{-\epsilon}\right) .
$$

Using this result we can prove the theorem for nonintegral values of $\gamma>-1$. If we set

$$
\tau_{R}^{\eta}=\eta \int_{0}^{R} S(r)(R-r)^{\eta-1} d r, \quad \eta>0,
$$

then we have $\bar{\sigma}_{R}=\tau_{R}^{\eta} / R^{\eta}$ and also

$$
\tau_{R}^{(\alpha+\beta)}=\frac{\Gamma(\alpha+\beta+1)}{\Gamma(\alpha+1) \Gamma(\beta)} \int_{0}^{R} \tau^{\alpha}(r)(R-r)^{\beta-1} d r, \quad \alpha \geqq 0, \beta>0 .
$$

For a proof of (14) see [3, p. 27, Lemma 6]. In view of (12) we must show that

$$
\tau_{R}^{\gamma+1} / R^{\gamma+1}=o(1)
$$

Using(14) we have

(4) It should be noted that for some $\xi$ the situation will be slightly better than in sum $A+B$. However, if $\tau(\xi)=0$ the situa tion is essentially that in $A+B$ and no better. 


$$
\frac{\tau_{R}^{\gamma+1}}{R^{\gamma+1}}=\frac{\tau_{R}^{k+(1-\epsilon)}}{R^{k+1-\epsilon}}=\frac{D}{R^{k+1-\epsilon}} \int_{0}^{R} \tau^{k}(r)(R-r)^{-\epsilon} d r
$$

where $D$ is a constant. We now break up this integral into two integrals $I_{1}$, extended from 0 to $R-1$, and $I_{2}$, taken from $R-1$ to $R$. To show that $I_{1}$ is $o(1)$ we integrate by parts and get

$$
\begin{aligned}
I_{1}=\frac{D}{R^{k+1-\epsilon}} & {\left[\int_{0}^{R-1} \tau^{k}(r) d r\right.} \\
& \left.\quad-\epsilon\left(\int_{0}^{R / 2}+\int_{R / 2}^{R-1}\right) \frac{1}{(R-r)^{1+\epsilon}}\left\{\int_{0}^{r} \tau^{k}(t) d t\right\} d r\right] .
\end{aligned}
$$

Taking into account (12) and (14), we may write

$$
I_{1}=o(1)+O\left(R^{-\epsilon}\right)+o(1) \int_{R / 2}^{R-1} \frac{d r}{(R-r)^{1+\epsilon}}=o(1) .
$$

It remains to show that $I_{2}=o(1)$, and for this we require the following result.

Lemma 5. Under the hypotheses of Theorem $2, \bar{\sigma}_{R}^{\lambda}=o\left(R^{\gamma+1-\lambda}\right), \lambda=0,1,2$, $\cdots, k$, where $k-1=[\gamma]$.

We decompose $\sigma_{R}^{\lambda}$ into sums $A^{\prime}$ and $B^{\prime}$ as in (8). As there, $B^{\prime}$ is $O\left(1 / R^{1+\zeta}\right)$, $\zeta=2(\bar{\gamma}+1)-\gamma$. To treat $A^{\prime}$ we proceed as in Lemma 4 and reduce the problem to a consideration of sums which are essentially $A$ and $B$ of (6), but with $a_{M}=o\left(|M|^{\gamma-\lambda}\right)$. The proofs of Theorem 1 and Lemma 4 show these sums to be $o\left(R^{\gamma+1-\lambda}\right)$. Hence $\sigma_{R}^{\lambda}$ is $o\left(R^{\gamma+1-\lambda}\right)$ and so $\bar{\sigma}_{R}^{\lambda}=o\left(R^{\gamma+1-\lambda}\right)$. The equivalence of the order conditions again follows from the proof given in Hardy [2]. In passing we note that the condition $a_{M}=o\left(|M|^{\gamma-\lambda}\right)$ is the worst one that can occur.

We now take up the evaluation of $I_{2}$.

$$
\begin{aligned}
I_{2}= & \frac{D}{R^{k+1-\epsilon}} \int_{R-1}^{R} \tau^{k}(r)(R-r)^{-\epsilon} d r \\
= & \frac{D}{R^{k+1-\epsilon}} \int_{R-1}^{R}\left\{\sum_{|M| \leqq r} A_{M}(r-|M|)^{k}\right\}(R-r)^{-\epsilon} d r \\
= & \frac{D}{R^{k+1-\epsilon}} \int_{R-1}^{R}\left\{\sum_{|M| \leqq R-1} A_{M}(r-|M|)^{k}\right\}(R-r)^{-\epsilon} d r \\
& +\frac{D}{R^{k+1-\epsilon}} \int_{R \rightarrow 1}^{R}\left\{\sum_{R-1<|M| \leqq r} A_{M}(r-|M|)^{k}\right\}(R-r)^{-\epsilon} d r \\
= & I_{2}^{\prime}+I_{2}^{\prime \prime} .
\end{aligned}
$$


Integrating $I_{2}^{\prime}$ by parts $k$ times gives

$$
\begin{aligned}
I_{2}^{\prime}= & \frac{D_{1}^{\prime}}{R^{k+1-\epsilon}} \sum_{|M| \leqq R-1} A_{M}(R-1-|M|)^{k} \\
& +\frac{D_{2}^{\prime}}{R^{k+1-\epsilon}} \sum_{|M| \leqq R-1} A_{M}(R-1-|M|)^{k-1}+\cdots \\
& +\frac{D_{k}^{\prime}}{R^{k+1-\epsilon}} \sum_{|M| \leqq R-1} A_{M}(R-1-|M|) \\
& -\frac{D_{k+1}^{\prime}}{R^{k+1-\epsilon}} \int_{R-1}^{R}\left\{\sum_{|M| \leqq R-1} A_{M}\right\}(R-r)^{k-\epsilon} d r \\
= & \left.\frac{D_{1}^{\prime}}{R^{k+1-\epsilon}} \tau^{k}(R-1)+\frac{D_{2}^{\prime}}{R^{k+1-\epsilon}} \tau^{(k-1)}+R-1\right) \cdots+\frac{D_{k+1}^{\prime} S(R-1)}{R^{k+1-\epsilon}}
\end{aligned}
$$

where $D_{1}^{\prime}, D_{2}^{\prime}, \cdots, D_{k+1}^{\prime}$ are constants. Lemma 5 tells us that the last sum is $o(1)$ and so $I_{2}^{\prime}=o(1)$. We now dispose of $I_{2}^{\prime \prime}$. This time we again integrate by parts $k$ times and get

$$
\begin{aligned}
I_{2}^{\prime \prime} & =D_{2}^{\prime \prime} \sum_{(R-1)<|M| \leqq R} A_{M}\left(1-\frac{|M|}{R}\right)^{k+1-\epsilon} \\
& =D_{2}^{\prime \prime} \sum_{P} a_{P} \sum_{(R-1)<|M| \leqq R} \alpha_{M-P}\left(1-\frac{|M|}{R}\right)^{k+1-\epsilon} \\
& \leqq D_{2}^{\prime \prime} \sum_{P}\left|a_{P}\right| \sum_{(R \rightarrow 1)<|M| \leqq R}\left|\alpha_{M-P}\right|\left|1-\frac{(R-1)}{R}\right|^{k+1-\epsilon} \\
& \leqq D_{2}^{\prime \prime}\left(\sum_{|P|<3 R / 2}+\sum_{|P| \geqq 3 R / 2}\right) \frac{\left|a_{P}\right|}{R^{k+1-\epsilon}} \sum_{(R-1)<|M| \leqq R}\left|\alpha_{M-P}\right|=A^{\prime \prime}+B^{\prime \prime} .
\end{aligned}
$$

The proof of the fact that $B^{\prime}$ in (8) is $o(1)$ and Remark 3 tell us that $B^{\prime \prime}$ is $o(1)$. From the proof of Lemma 3 it is easily seen that

$$
\sum_{(R-1)<|M| \leqq R}\left|\alpha_{M-P}\right|=\left\{\begin{array}{l}
O(1) \text { if } R-1 \leqq|P| \leqq R+1 \\
O\left(\frac{1}{|| P|-R|^{3+2(\bar{\gamma}+1)}}\right) \text { otherwise. }
\end{array}\right.
$$

Also we have that $\left|a_{P}\right| / R^{k+1-\epsilon}=o\left(|P|^{k-\epsilon} / R^{k+1-\epsilon}\right)$. This plus the fact that, in sum $A^{\prime \prime},|P|<3 R / 2$ show that the consideration of $A^{\prime \prime}$ reduces to consideration of sums essentially the same as $A$ and $B$ of (6). These we know are $o(1)$, and so the proof of the present theorem is completed.

Corollary. In the event that the series $T_{1}$ is a Fourier series of a function 
in $L_{p}, 1<p \leqq 2$, then $T_{3}$ is circularly summable $(C, 1 / p)$ to zero, uniformly on $E$.

We first prove the following lemma.

Lemma 6. Let $F(x, y)$ be in $L_{p}, 1<p \leqq 2$, periodic of period $2 \pi$ in each variable, and suppose $F \sim \sum a_{M} e^{i M X}$. Then

$$
\sum_{(R-1)<|M|<R}\left|a_{M}\right|=o\left(R^{1 / p}\right)
$$

By the Cauchy-Schwarz inequality

$$
\sum_{(R-1)<|M|<R}\left|a_{M}\right| \leqq\left(\sum_{R \rightarrow 1<|M|<R}\left|a_{M}\right|^{p^{\prime}}\right)^{1 / p^{\prime}}\left(L_{(R-1, R)}\right)^{1 / p},
$$

where $1 / p+1 / p^{\prime}=1$ and $L_{(R-1, R)}$ denotes the number of lattice points in the ring whose radii are $R-1$ and $R$. From (2) and the theorem of HausdorffYoung we see immediately that $(15)$ is $o\left(R^{1 / p}\right)$.

To prove the corollary we first note that the Riemann-Lebesgue theorem tells us that $a_{M}=o(1)$ as $|M| \rightarrow \infty$. By making a few minor changes in the proof of Theorem 2 for the case $\gamma=0$, we can show that the formal product is $(C, 1)$ summable to zero and that $\sigma_{R}^{(1)}=o\left(R^{1-1 / p}\right)$. From part two of Theorem 2 we then conclude that the formal product is summable $(C, 1 / p)$ to zero.

We also have the following counterpart of Theorem $1^{\prime}$.

TheOREM 2'. Let $T_{1}$ and $T_{2}$ be two series with coefficients as described in Theorem 2. Further, let

$$
\frac{\partial^{(r)}}{\partial x^{(r)}} \frac{\partial^{(s)}}{\partial y^{(8)}}\left(\nabla^{2}\right)^{(t)} T_{2}=0
$$

for all triples of integers $(r, s, t)$ such that $0<r+s+t \leqq \gamma+1$, uniformly for all $(x, y)$ in a set $E \subset \Omega$, where $\Omega$ is the fundamental square. Suppose $T_{2}$ converges to a function $\lambda(x, y)$ on $\Omega$, where $\lambda(x, y)$ need not be zero on $E$, but

$$
\frac{\partial^{(r)}}{\partial x^{(r)}} \frac{\partial^{(s)}}{\partial y^{(s)}}\left(\nabla^{2}\right)^{(t)} \lambda(x, y)=0
$$

on $E$ for all triples of integers $(r, s, t)$ such that $0<r+s+t \leqq \gamma+1$. Then the series

$$
T_{3}-\lambda T_{1}
$$

is circularly summable $(C, \gamma+1)$ to zero, uniformly for $(x, y)$ in $E\left(^{5}\right)$.

The proof is similar to that of Theorem $1^{\prime}$, but with Theorem 2 now playing the role of Theorem 1 .

${ }^{(5)}$ Examples of series $T_{2}$ and functions $\lambda(x, y)$ satisfying such conditions are the localizing functions and their Fourier series which are described in the next section. 
3. Localization. The above results will now be applied to the problem of localization. However, before doing so, a few preliminary remarks are in order.

REMARK 5. If $f(x, y)$ is in $L$ and periodic of period $2 \pi$ in each variable then, as is well known, we may associate to $f$ its Fourier series. Thus

$$
f \sim \sum c_{M} e^{i M X}=\subseteq[f] .
$$

In what follows the notation $\subseteq[f]$ will be used quite frequently to denote the Fourier series of a function $f$. The circular partial sums $S_{R}(x, y)$ of $f$ are given by

$$
\begin{aligned}
S_{R}(x, y) & =\sum_{|M| \leqq R} c_{M} e^{i M X}=\sum_{|M| \leqq R}\left[\frac{1}{4 \pi^{2}} \int_{0}^{2 \pi} \int_{0}^{2 \pi} f(u, v) e^{-i(m u+n v)} d u d v\right] e^{i M X} \\
& =\frac{1}{4 \pi^{2}} \int_{0}^{2 \pi} \int_{0}^{2 \pi} f(u, v) D_{R}(u-x, v-y) d u d v
\end{aligned}
$$

where

$$
D_{R}(x, y)=\sum_{|M| \leqq R} e^{-i M X}=\sum_{|M| \leqq R} e^{i M X}=D_{R}(-x,-y) .
$$

The function $D_{R}(x, y)$ is thus a sort of Dirichlet kernel for circular summation.

Remark 6. Let $T$ be a double trigonometric series, $T=\sum c_{M} e^{i M x}$. An operator $\nabla^{-2}$, which shall be called the anti-Laplacian operator, is defined to act on $T$ as follows.

$$
\nabla^{-2} T=\frac{c_{00}(x+y)^{2}}{4}+\sum_{|M| \neq 0} \frac{-c_{M}}{|M|^{2}} e^{i M X} .
$$

That is, $\nabla^{-2}$ is a formal right inverse to the Laplacian. Note that $\nabla^{2}\left(\nabla^{-2}\right) T$ $=T$ always, but that $\left(\nabla^{-2}\right) \nabla^{2} T \neq T$ if $c_{00} \neq 0$. In this train, we define the $k$ th power of the anti-Laplacian to be the formal right inverse of $\left(\nabla^{2}\right)^{(k)}$. Explicitly

$$
\left(\nabla^{-2}\right)^{(k)} T=\frac{c_{00}(x+y)^{2 k}}{2^{k}[(2 k) !]}+\sum_{|M| \neq 0} \frac{(-1)^{k} c_{M}}{|M|^{2 k}} e^{i M X} .
$$

If $c_{M}=o(|M| \gamma), \gamma \geqq-1$, then $\left(\nabla^{-2}\right)^{[\gamma / 2]+2} T$ will converge uniformly, either rapidly or circularly, to some function $F(x, y)$. This is true by virtue of the fact that in this event $c_{M} /|M|^{2 k}=o\left(\left(|M|^{2}\right)^{\gamma / 2-[\gamma / 2]-2}\right)$ and so $\sum\left|c_{M}\right| /|M|^{2 k}<\infty$. If $c_{00}=0$, the Riesz-Fischer Theorem tells us that $\left(\nabla^{-2}\right)^{([r / 2]+2)} T$ is $\subseteq[f]$.

REMARK 7. We shall now discuss another essential notion, that of localizing function.

By a domain we shall, as usual, mean an open connected set. When speaking of a closed domain we shall mean a domain plus its boundary. Now let 
$\Re$ be a closed domain contained in the interior of the fundamental square $\Omega$, let $\Re^{0}$ denote the interior of $\Re$, and let $\Re^{\prime}$ be another closed domain such that $\Re^{\prime} \subset \Re^{0}$. A function $\lambda(x, y)$ which is continuous, is of period $2 \pi$ in each variable, has Fourier coefficients $O\left(|M|^{-\mu}\right), \mu$ a sufficiently large positive integer, and such that $\lambda(x, y)=0$ for $(x, y)$ not in $\Re(\bmod 2 \pi)$ and $\lambda(x, y)=1$ for $(x, y)$ in $\Re^{\prime}(\bmod 2 \pi)$, is called a localizing function for the domains $\Re$ and $\mathfrak{R}^{\prime}$.

To obtain $\lambda(x, y)$ we proceed as follows. Let $\Re_{1}$ be a closed domain such that $\Re^{\prime}$ is interior to $\Re_{1}, \Re_{1} \subset \Re^{0}$, and all boundary points of $\Re_{1}$ are at distance $\epsilon$ from the boundary of $\Re$, where $\epsilon$ is less than one half the distance from the boundary of $\Re$ to that of $\Re^{\prime}$. Let $K(s, t)$ be a function of class $C^{(\mu)}$ which is zero outside of a circle $C$ with center at the origin and radius $\epsilon$ and such that the integral of $K(s, t)$ taken over $C$ is one. Finally, let $\phi(x, y)$ be a function which is one on $\Re_{1}$ and zero elsewhere. Then it is easily verified that the function

$$
\lambda(x, y)=\iint_{C(x, y ; \epsilon)} \phi(s, t) K(s-x, t-x) d s d t,
$$

where $C(x, y ; \epsilon)$ is the circle with center at $(x, y)$ and radius $\epsilon$, is a localizing function for $\Re$ and $\Re^{\prime}$. This construction is due to Dr. A. Calderón. If $\Re$ and $\Re^{\prime}$ are rectangles or concentric circles, simpler constructions are available.

We now state the theorem from which we deduce our localization theorem.

THEOREM 3. Let $T_{1}$ be a double trigonometric series with coefficients $a_{M}$ $=o\left(|M|^{\gamma}\right), \gamma \geqq-1$. Then the series $\left(\nabla^{-2}\right)^{(\omega)} T_{1}$ with $\omega=[\gamma / 2]+2$ converges uniformly to a function $F(x, y)$. Further, let $\lambda(x, y)$ be a localizing function of class $C^{(2(\delta+2 \omega))}$ associated with the domains $\Re$ and $\Re^{\prime}$ and whose Fourier coeffcients are consequently $\alpha_{M}=O\left(|M|^{-(\delta+2 \omega)}\right)$, where once again $\delta=5+2(\bar{\gamma}+1)$. Then the difference

$$
\begin{aligned}
\Delta_{R}(x, y)= & \sum_{|M| \leqq R} a_{M} e^{i M X} \\
& -\frac{1}{4 \pi^{2}} \int_{0}^{2 \pi} \int_{0}^{2 \pi} F(u, v) \lambda(u, v)\left(\nabla^{2}\right)^{(\omega)} D_{R}(u-x, v-y) d u d v
\end{aligned}
$$

is summable $(C, \gamma+1)$ to zero, uniformly for all $(x, y)$ in $\Re^{\prime}$.

If $T_{1}$ is a Fourier series of a function $f$ in $L_{p}, 1<p \leqq 2$, then $\Delta_{R}(x, y)$ is summable $(C, 1 / p)$ to zero.

The uniform convergence of $\left(\nabla^{-2}\right)^{(\omega)} T_{1}$ to $F(x, y)$ was shown in Remark 6. In the rest of the proof we consider two cases. First, suppose that $a_{00}=0$. Then, as has already been noted, $\left(\nabla^{-2}\right)^{(\omega)} T_{1}$ is $\subseteq[F]$ and converges absolutely and uniformly to $F$. Also, $\subseteq[\lambda]$ converges absolutely and uniformly to $\lambda$. Thus the formal product $\subseteq[F] \subseteq[\lambda]$ is $\subseteq[F \lambda]$. Now clearly, the function $\Delta_{R}(x, y)$ is the circular partial sum of rank $R$ of the series $T_{1}-\left(\nabla^{2}\right)^{(\omega)}\{\Im[F \lambda]\}$. 
But in view of the above discussion,

$$
T_{1}-\left(\nabla^{2}\right)^{(\omega)}\{\subseteq[F \lambda]\}=T_{1}-\nabla^{2}(\omega)\{\subseteq[F] \subseteq[\lambda]\} .
$$

Utilizing (9), we can write this as

$$
\begin{aligned}
T_{1}-\left(\nabla^{2}\right)^{(\omega-1)}\left[\left\{\nabla^{2} \subseteq[F]\right\} \subseteq[\lambda]+\right. & 2\left(\frac{\partial \subseteq[F]}{\partial x} \frac{\partial \subseteq[\lambda]}{\partial x}\right. \\
& \left.\left.+\frac{\partial \subseteq[F]}{\partial y} \frac{\partial \subseteq[\lambda]}{\partial y}\right)+\subseteq[F]\left\{\nabla^{2} \subseteq[\lambda]\right\}\right] .
\end{aligned}
$$

It is clear that repeated applications will therefore yield

$$
\begin{aligned}
&\left(\nabla^{2}\right)^{\omega}\{\subseteq[F \lambda]\}=\sum_{r, s, t, u} \beta_{r, s, t, u}\left\{\left(\nabla^{2}\right)^{(r)} \frac{\partial^{(s)}}{\partial x^{(s)}} \frac{\partial^{(t)}}{\partial y^{(t)}} \subseteq[F]\right\} \\
& \cdot\left\{\left(\nabla^{2}\right)^{(u)} \frac{\partial^{(s)}}{\partial x^{(s)}} \frac{\partial^{(t)}}{\partial y^{(t)}} \subseteq[\lambda]\right\}
\end{aligned}
$$

where the numbers $\beta_{r, s, t, u}$ are constants and the summation is taken over all quadruples of integers $(r, s, t, u)$ such that $2 r+s+t+2 u=2 \omega$. Thus

$$
\begin{aligned}
T_{1}-\left(\nabla^{2}\right)^{(\omega)} \subseteq[F \lambda] & =T_{1}-\left\{\left(\nabla^{2}\right)^{(\omega)} \subseteq[F]\right\} \subseteq[\lambda]-\sum_{r \neq \omega} \beta_{r, s, t, u}\{\}\{\} \\
& =T_{1}(1-\subseteq[\lambda])-\sum_{r \neq \omega} \beta_{r, s, t, u}\{\}\{\}
\end{aligned}
$$

since $\subseteq[F]=\left(\nabla^{-2}\right)^{(\omega)} T_{1}$. Each of the series

$$
1-\subseteq[\lambda], \quad\left(\nabla^{2}\right)^{(u)} \frac{\partial^{(s)}}{\partial x^{(s)}} \frac{\partial^{(t)}}{\partial y^{(t)}} \subseteq[\lambda]
$$

is zero in $\Re^{\prime}$, satisfies equation (7), and has coefficients $\alpha_{M}=O\left(|M|^{-\delta}\right)$. On the other hand, each of the series

$$
T_{1}, \quad\left(\nabla^{2}\right)^{(r)} \frac{\partial^{(s)}}{\partial x^{(s)}} \frac{\partial^{(t)}}{\partial y^{(t)}} \subseteq[F]
$$

has coefficients at worst $o\left(|M|^{\gamma}\right)$. Therefore, Theorem 2 is applicable to each of the terms in (17), and so each series is summable $(C, \gamma+1)$ to zero, uniformly in $\Re^{\prime}$.

In case two we consider series $T_{1}$ which consist only of the constant term $a_{00}$. Then equation (16) reads

$$
\Delta_{R}(x, y)=a_{00}-\frac{1}{4 \pi^{2}} \int_{0}^{2 \pi} \int_{0}^{2 \pi} F(u, v) \lambda(u, v)\left(\nabla^{2}\right)^{(\omega)} D_{R}(u-x, v-y) d u d v .
$$

Integrating by parts $2 \omega$ times gives 


$$
\Delta_{R}(x, y)=a_{00}-\frac{1}{4 \pi^{2}} \int_{0}^{2 \pi} \int_{0}^{2 \pi}\left(\nabla^{2}\right)^{(\omega)}\{F(u, v) \lambda(u, v)\} D_{R}(u-x, v-y) d u d v
$$

If we define the function $\Gamma(u, v)$ to be $\left(\nabla^{2}\right)^{(\omega)}\{F(u, v) \lambda(u, v)\}$ for $0 \leqq u \leqq 2 \pi$, $0 \leqq v \leqq 2 \pi$ and extend the function to be periodic of period $2 \pi$ in each variable, then the integral in (18) is merely the circular partial sum of rank $R$ of the Fourier series of $\Gamma$ at the point $(x, y)$. Now

$$
\begin{aligned}
\Gamma(u, v) & =\left(\nabla^{2}\right)^{(\omega)}\left[\frac{a_{00}(u+v)^{2 \omega} \lambda(u, v)}{2^{\omega}[(2 \omega) !]}\right] \\
& =a_{00} \sum_{r+s+t=2 \omega} d_{r, s, t}(u+v)^{(2 \omega-r)} \frac{\partial^{(s+t)}}{\partial u^{(s)} \partial v^{(t)}} \lambda(u, v)
\end{aligned}
$$

where $r, s, t$, are positive integers or zero. Recalling that $\lambda(u, v)$ has Fourier coefficients $O\left(|M|^{-(\delta+2 \omega)}\right)$ and is zero outside of $\Re$, we see that $\Gamma(u, v)$ is continuous and has an absolutely and uniformly convergent Fourier series in the fundamental square $\Omega$. From the uniformity of the convergence and the continuity of $\Gamma$ we learn that $\mathfrak{S}[\Gamma]$ must converge to $\Gamma$ at every point. From the absolute convergence we find that the series must be absolutely circularly summable and therefore uniformly circularly summable. In summary, the integral in (18) tends to $\Gamma(x, y)$ as $R$ tends to infinity, uniformly for all $(x, y)$ in $\Omega$. But for $(x, y)$ in $\Re^{\prime}, \Gamma(x, y)=a_{00}$, as can be seen from (19). Consequently, $\Delta_{R}(x, y) \rightarrow 0$ as $R \rightarrow \infty$, uniformly for all $(x, y)$ in $\Re^{\prime}$.

Since any series $T_{1}$ is the sum of two series, one consisting of the constant term alone and the other of the nonconstant terms, we combine the results of the two cases just treated to establish (16).

In the event that $T_{1}$ is a Fourier series of a function in $L_{p}, 1<p \leqq 2$, the proof of the general case still applies. However, the corollary, rather than Theorem 2, is used in the appropriate place.

REMARK 8. If in (19) we replace the function $a_{00}(u+v)^{2 \omega} / 2 \omega[(2 \omega)$ !] by any function $H(u, v)$ which has all derivatives of order $2(\omega+2)$ on $\Re^{0}$, which has the property that it and its derivatives remain bounded as the boundary of $\Re$ is approached, and which satisfies the partial differential equation $\left(\nabla^{2}\right)^{(\omega)} H=0$ on $\Re^{0}$, then we can modify the above discussion very slightly to show that

$$
\frac{1}{4 \pi^{2}} \int_{0}^{2 \pi} \int_{0}^{2 \pi} F(u, v) \lambda(u, v)\left(\nabla^{2}\right)^{(\omega)} D_{R}(u-x, v-y) d u d v \rightarrow 0
$$

as $R \rightarrow \infty$, uniformly for $(x, y)$ in $\Re^{\prime}$.

THEOREM 4. If $T$ and $T^{\prime}$ are two double trigonometric series with coefficients $o\left(|M|^{\gamma}\right), \gamma \geqq-1$, and if the functions $F$ and $F^{\prime}$ associated with each of these series are equal in a closed domain $\Re$ contained in the interior of the fundamental 
square $\Omega$, then in every smaller closed domain $\Re^{\prime}$ contained in $\Re^{0}$, the interior of $\Re$, the series $T-T^{\prime}$ is uniformly summable $(C, \gamma+1)$ to zero. The condition that $F-F^{\prime}$ vanish in $\Re$ can be replaced by a more general one. For example, if $\gamma=-1$, then if $F$ and $F^{\prime}$ differ in $\Re$ by a function $G$ which has all derivatives of order six in $\Re^{0}$, is harmonic in $\Re^{0}$, and which has the property that its derivatives are bounded as the boundary of $\Re$ is approached, we find that the series $T-T^{\prime}$ will still be summable to zero, uniformly for $(x, y)$ in $\mathfrak{R}^{\prime}$. If $\gamma>-1$ and $G$ has all derivatives of order $2(\omega+2)$ in $\Re^{0}$, satisfies the partial differential equation $\left(\nabla^{2}\right)^{(\omega)} G=0$ there, and fulfills the requirement that its derivatives remain bounded as the boundary is approached, then we have the series $T-T^{\prime}$ uniformly summable $(C, \gamma+1)$ to zero on $\Re^{\prime}$.

If the series are Fourier series of functions $f$ and $f^{\prime}$ in $L_{p}, 1<p \leqq 2$, we get that $T-T^{\prime}$ is summable $(C, 1 / p)$ to zero, uniformly on $\Re^{\prime}$.

All of the above statements are immediate consequences of the preceding theorem, with the generalizations following from Remark 8.

4. The conjugate series. By a series conjugate to a double trigonometric series $T=\sum c_{M} e^{i M X}$, we shall mean the double trigonometric series $\tilde{T}$ given by

$$
\tilde{T}=\sum \epsilon_{m n} c_{m n} e^{i(m x+n y)}
$$

where $\epsilon_{m n}=\operatorname{signum}(m n)$. For a discussion of this definition see [5]. On the basis of results in one dimension [6] one would expect the following theorem to hold.

"Let $\tilde{T}$ and $\tilde{T}^{\prime}$ be series conjugate to the series $T$ and $T^{\prime}$ of Theorem 4. Then under the conditions of that theorem, the conclusions apply to the series $\widetilde{T}$ and $\widetilde{T}^{\prime}$, but with the words 'summable to zero' replaced by 'summable'."

In proving such a result by our methods, the key theorem would be the one on multiplication which would now read:

"Let $T_{1}$ and $T_{2}$ be two series satisfying the hypotheses of Theorem 2 . Then the series $\tilde{T}_{3}$ conjugate to the formal product of $T_{1}$ and $T_{2}$ is uniformly summable $(C, \gamma+1)$ on the set $E . "$

This statement, surprisingly enough, is false, as the following simple example shows. Let $T_{1}=\sum a_{m n} \exp i(m x+n y)$, where

$$
a_{m n}=\left\{\begin{array}{l}
1 / m \log (m+1), \text { if } n=0, m \geqq 1, \\
0, \quad \text { otherwise. }
\end{array}\right.
$$

Let $T_{2}=2 i \sin (x+y)=\exp i(x+y)-\exp i(x+y)$, and take the set $E$ to be the origin. Hence $a_{m n}=o\left(1 /\left(m^{2}+n^{2}\right)^{1 / 2}\right)$ and $T_{2}=0$ on $E$. The formal product $T_{3}$ is given by

$$
T_{3}=\sum A_{m e n}, \quad A_{m n}=a_{m-1, n-1}-a_{m+1, n+1} .
$$

Thus all $A_{m n}=0$ except the following 
$A_{m, 1}=1 /(m-1) \log m$ if $m \geqq 2, A_{m,-1}=-1 /(m+1) \log (m+2)$ if $m \geqq 0$.

Clearly, the series $T_{3}$ is circularly summable to 0 at the origin, while $\tilde{T}_{3}$ diverges to $+\infty$. Thus $\tilde{T}_{3}$ is summable by no regular method. As a matter of fact we can take for $T_{2}$ a function with a zero at the origin of arbitrarily large order and the conclusion will still hold. For example, take $\sin ^{2 n+1}(x+y)$, $n=0,1,2, \cdots$.

5. Extensions and generalizations. In this section we shall generalize our results in two distinct directions. First, we extend our results to the $k$-dimensional case.

If we make the obvious changes in our definitions and notation to conform to the $k$-dimensional situation, we have the following localization theorem.

THEOREM 5. Let $T$ and $T^{\prime}$ be two $k$-dimensional trigonometric series, $k \geqq 1$, with coefficients $o\left(|M|^{\gamma}\right), \gamma \geqq-(k-1)$. Let $\left(\nabla^{-2}\right)^{(\omega)} T=F$ and $\left(\nabla^{-2}\right)^{(\omega)} T^{\prime}=F^{\prime}$, where now $\omega=[\gamma / 2]+[k / 2]+1$. If $F$ and $F^{\prime}$ are equal in a closed domain $\Re$ contained in the interior of the fundamental k-dimensional cube $\Omega$, the series $T-T^{\prime}$ is spherically summable $(C, \gamma+k-1)$ to zero, uniformly on every closed domain $\mathfrak{R}^{\prime}$ contained in the interior of $\Re$.

REMARK 9. As in Theorem 4 the condition $F-F^{\prime}=0$ on $\Re$ can be replaced by a more general one. We leave its formulation to the reader.

An examination of the proof for $k=2$ shows that with proper modifications of orders of coefficients and of the exponents of the various operators, the proof is valid for all $k \geqq 1$. For $k=1$, however, these results are not new, having been obtained by Riemann, Rajchman, and Zygmund. The reader is referred to [7] where complete references for the case $k=1$ are listed.

The second direction in which we generalize some of our results is that of considering the sums $S_{R}$ defined by certain curves other than circles. In keeping with our former notation we shall denote a point $(u, v)$ in the plane merely by $U$. For every $\beta \geqq 1$ we can introduce the $L_{\beta}$ norm into the plane as follows. Denoting the $L_{\beta}$ norm of $U$ by $|U|_{\beta}$, we define

$$
|U|_{\beta}=\left(|u|^{\beta}+|v|^{\beta}\right)^{1 / \beta}
$$

Consider now a curve $C_{R, \beta}$ which is symmetric with respect to the coordinate axes and whose equation in the first quadrant is

$$
x^{\beta}+y^{\beta}=R^{\beta} \text {, }
$$

Clearly, the curves $C_{R, \beta}$ are circles with center at origin and radius $R$ in the $L_{\beta}$ metric. (For $\beta=1$ we get diamonds, for $\beta=2$, circles.) For brevity we shall call curves which are circles in the $L_{\beta}$ norm $L_{\beta}$ circles.

Let $T=\sum c_{M} \exp (i M X)$ be a double trigonometric series. We define the type $\beta$ circular partial sums of rank $R$ of $T$ by the equation

$$
S_{R, \beta}(x, y)=\sum_{|M| \beta \leqq R} c_{M} e^{i M X}, \quad \beta \geqq 1 .
$$


If $\beta=2$ we obtain the ordinary circular partial sums which we have already considered. For other values of $\beta$ the numbers $S_{R, \beta}(x, y)$ are obtained by summing those terms of $T$ whose indices lie within or on the curve $C_{R, \beta}$. We shall say that $T$ is $\beta$ circularly summable to $L(x, y)$ for $(x, y)$ in a set $E$ in case $\lim _{R \rightarrow \infty} S_{R, \beta}(x, y)=L(x, y)$.

We now state the following generalization of Theorem 1.

THEOREM 6. Let $T_{1}$ and $T_{2}$ be two double trigonometric series with the following properties

$$
a_{M}=o\left(|M|_{\beta}^{-1}\right)
$$

$$
\alpha_{M}=O\left(|M|_{\beta}^{-5}\right), \quad \beta>1,
$$

$$
\sum \alpha_{M} e^{i M X}=0,
$$

for $(x, y)$ belonging to a set $E$ in the plane. Then the series $T_{3}$, whose terms $A_{M}$ are given by (1), is the formal product of $T_{1}$ and $T_{2}$ and is $\beta$ circularly summable to zero, uniformly for all $(x, y)$ in $E$.

To prove the theorem we first notice that the number of lattice points contained within and on a curve $C_{R, \beta}$ is $K(\beta) R^{2}+O(R)$, where $K(\beta)$ is a constant which varies with $\beta$. With this observation taking the place of Lemma 2, we can go through the proofs of Lemma 1, Lemma 3, and Theorem 1 , replace the symbols $|M|,|P|$, and $S_{R}$ by $|M|_{\beta},|P|_{\beta}$, and $S_{R, \beta}$ respectively, and interpret the words "circle" and "annulus" to mean " $L_{\beta}$ circle" and "annulus in the $L_{\beta}$ norm."

We now state a theorem which generalizes part of Theorem 2 and contains the localization principle for $\beta$ circular summability of double trigonometric series with sufficiently good coefficients.

THEOREM 7. Let $T_{1}$ be a double trigonometric series with coefficients $a_{M}$ $=o\left(|M|_{\beta}^{-1}\right), \beta \geqq 1$. Then the series $\left(\nabla^{-2}\right) T_{1}$ converges uniformly to a function $F(x, y)$. Let $\lambda(x, y)$ be a localizing function associated with domains $\Re$ and $\Re^{\prime}$. If $\lambda(x, y)$ has Fourier coefficients $\alpha_{M}=O\left(|M|_{\beta}^{-5}\right)$, then the difference

$$
\begin{aligned}
\Delta_{R, \beta}(x, y)= & \sum_{|M| \beta \leqq R} a_{M} e^{i M X} \\
& -\frac{1}{4 \pi^{2}} \int_{0}^{2 \pi} \int_{0}^{2 \pi} F(u, v) \lambda(u, v)\left(\nabla^{2}\right) D_{R, \beta}(u-x, v-y) d u d v,
\end{aligned}
$$

where $D_{R, \beta}(x, y)=\sum_{|M|_{\beta} \leqq R} e^{i M X}$, tends to zero as $R \rightarrow \infty$, uniformly for all $(x, y)$ in $\Re^{\prime}$.

The theorem just stated is a rephrasing of Theorem 3 for the case $\gamma=-1$ and with $|M|$ replaced by $|M|_{\beta}$. Thus once we establish that $\left(\nabla^{-2}\right) T_{1}$ converges absolutely and hence uniformly to $F(x, y)$, the proof of Theorem 3 goes through with the proper modification of symbols and reinterpretation of certain statements for the case now being taken up. 
The coefficients of $\left(\nabla^{-2}\right) T_{1}$ are $o\left(1 /\left(|m|^{\beta}+|n|^{\beta}\right)^{1 / \beta}\left(|m|^{2}+|n|^{2}\right)\right)$. If $\beta \geqq 2$ then $1 /\left(|m|^{\beta}+|n|^{\beta}\right)^{1 / \beta} \leqq 1 /\left(|m|^{2}+|n|^{2}\right)^{1 / \beta}$, and so $\left(\nabla^{-2}\right) T_{1}$ is majorized by a series whose coefficients are $o\left(1 /\left(|m|^{2}+|n|^{2}\right)^{1+1 / \beta}\right)$. In the event that $1 \leqq \beta \leqq 2$, we have the following inequality which can be readily verified.

$$
1 /\left(|m|^{\beta}+|n| \beta\right)^{1 / \beta} \leqq 1 /\left(m^{2}+n^{2}\right)^{1 / 2}, \quad 1 \leqq \beta \leqq 2 .
$$

Hence in this case $\left(\nabla^{-2}\right) T_{1}$ is majorized by a series whose coefficients are $o\left(1 /\left(m^{2}+n^{2}\right)^{3 / 2}\right)$ and so is surely absolutely convergent. Theorem 7 is thus proved.

If we introduce the $L_{\infty}$ metric in which the norm of a point $U=(u, v)$ is defined by $|U|_{\infty}=\max (|u|,|v|)$, our curves $C_{R, \infty}$ would be squares with center at the origin and side $2 R$. Consideration of the partial sums $S_{R, \infty}(x, y)$ would reduce to a very special case of restricted convergence of series [4]. Since the consideration of localization problems for series summed in the restricted sense will be discussed in a forthcoming paper, we refrain from considering the $L_{\infty}$ norm here.

\section{BIBLIOGRAPHY}

1. S. Bochner, Summation of multiple Fourier series by spherical means, Trans. Amer. Math. Soc. vol. 40 (1936) pp. 175-207.

2. G. H. Hardy, The second theorem of consistency for summable series, Proc. London Math. Soc. (2) vol. 15 (1916) pp. 72-88.

3. G. H. Hardy and Marcel Riesz, The general theory of Dirichlet's series, Cambridge, 1915.

4. C. N. Moore, On the summability of double Fourier series of discontinuous functions, Math. Ann. vol. 74 (1913) pp. 555-572.

5. K. Sokol-Sokolowski, On trigonometric series conjugate to Fourier series in two variables, Fund. Math. vol. 34 (1947) pp. 166-182.

6. A. Zygmund, Sur la theorie riemannienne des series trigonometriques, Math. Zeit. vol. 24 (1926) pp. 47-104.

7. — , Trigonometrical series, Warsaw, 1935.

The University of Chicago,

Chicago, Ill. 